\title{
POLÍTICA AMBIENTAL: A UTILIZAÇÃO DE TAXAS PIGOUVIANAS NO CASO DINÂMICO*
}

\author{
Francisco S. Ramos**
}

Henry Tulkens***

\section{Resumo}

A adoção de instrumentos de política ambiental para a correção dos problemas causados pela poluição é, geralmente, abordada num contexto estático, em que o objetivo é a determinação do nível ótimo de uma variável de controle. No caso em que esta variável é a taxa, isto acarreta custos adicionais para as firmas, podendo forçálas a sair do mercado. Todavia, dado que há uma alteração nos preços relativos dos fatores, é de se esperar que ocorra um processo de substituição. Este trabalho mostra a viabilidade da adoção de uma política ambiontal em que a variável de controle é gradualmente modificada. Ele se baseia na determinação de um processóque, através de uma sucessão de estados realizávcis para a conomia, é capaz de condu\%i-la desde uma situação ineficiente até um estado ótimo, utilizando como mecanismo corretor as laxas Pigouvianas.

\section{Abstract}

'The adoption of instruments of environmental policy (to correct the problems posed by pollution) is approached generally in a static context. 'The objective is normally to find out the optimal level of a control variable. If this variable is represented by taxes, firms face additional costs, and can be forced to go out from the market.

* O primeiro autor agradece o suporte financeiro da CAPLS/CNPq, e ambos agradecem as sugestōes de dois pareceristas desta Revista.

** Departamento de Economia, Centro de Ciéncias Sociais Aplicadas, Universidade Féederal de Pernambuco.

*** Center for Operations Research \& L̇cononntrics, Université Catholique de I.ouvain, Bélgica.

R. de Econometria Rio de Janeiro v. $15, \mathrm{n}^{\mathbf{0}}$ 2, pp.87-105 Novembro/Narço 1996 
However, given that relative prices of inputs change, it is expected that occurs a process of substitution. In this paper, the objective is the determination of a dynamic process that, through a succession of feasible states to the economy, can bring it from an inefficient situation to an optimum state. To do this, Pigovian taxes are used.

Palavras-Chave: Taxas Pigouvianas, ajustamentos dinâmicos, análise de estabilidade Códligo JEL: D50, Q20, H21, H41

\section{Introdução.}

A literatura sobre os instrumentos de política ambiental é bastante vasta. Tais instrumentos variam desde o clássico "commandand-control" até a criação de mercados artificiais, passando pelas taxas à la Pigou. Cada um deles apresenta vantagens e desvantagens que dependem do contexto em que é adotado ${ }^{1}$.

A grande maioria dos trabalhos sobre política ambiental se situa num contexto estático: busca-se sempre determinar qual o nivel ótimo das emissões, qual a quantidade ótima de certificados de enissão de poluição, ou qual a taxa ótima sobre as emissões, por exemplo. Entretanto, a adoção de qualquer um destes instrumentos, se realizada de forma instantânea, pode forçar a saída de firmas do mercado. Este fato pode ocorrer dado que a firma passa a enfrentar novos custos (caso das taxas e dos certificados) ou a efetuar restrições quantitativas sobre o seu nível de produção (via limite sobre emissões). A sugestão é de que a utilização de tais instrumentos se faça de forma gradual, de modo que as firmas disponham de um período de tempo para se ajustar ao novo contexto, efetuando, assim, o processo de substituição decorrente da alteração nos preços relativos dos fatores, como no caso das taxas.

1 Ressalte-se que não é objetivo deste trabalho enumerar as vantagens e/ou desvantagens destes instrumentos. Uma análise comparativa é feita por Bohm e Russell (1985). 
A literatura econômica, desde as contribuições seminais de Malinvaud (1970-1972) e Drèze-de la Vallée Poussin (1971), registra vários trabalhos que tratam de ajustamentos dinãmicos envolvendo bens públicos. No que concerne às aplicações sobre meioambiente, tem-se o artigo de Tulkens e Schoumaker (1975) que utiliza a análise de estabilidade para obter informações relativas aos efeitos do princípio "poluidor-pagador" sobre o bem-estar dos agentes econômicos. Tulkens (1979) emprega esta mesma metodologia para analisar problemas de poluição transfronteira no Mar do Norte. Em 1992 Ramos inclui a produção neste tipo de modelo a fim de analisar políticas de "command-and-control" 2 . Entretanto, este tipo de política apresenta uma série de limitações, como por exemplo a ineficiência em termos de custos ${ }^{3}$.

Este trabalho é uma extensão de Ramos (1992), e tem por objetivo apresentar um modelo de uma economia com poluição em que se usa um instrumento incitativo - as taxas Pigouvianas - de forma gradual, ou seja, a taxa é aumentada desde zero (a situação inicial) até atingir um valor considerado ótimo. Neste sentido, apresenta-se na Seção 2 o modelo da economia com poluição, onde se estabelece as condições para uma alocação eficiente no sentido de Pareto ${ }^{4}$. Em seguida, mostra-se que a utilização de taxas Pigouvianas permite atingir tal alocação. Por fim, se estabelece um processo dinâmico em que se descreve a evolução da economia desde uma situação não-ótima, através de uma sucessão de estados realizáveis, até uma situação eficiente. As propriedades do processo são então analisadas.

2 Uma excelente revisão da literatura sobre processos dinämicos pode ser vista em Tulkens (1978) e Chander e Parikh (1990).

3 Ver Baurnol e Oates (1988) para demonstração.

4 Confornx será visto, o nodelodifere daģueles usados nos livros-texto, como Baumol e Oates (1988), devido à forma particular da função de produção. 


\section{Especificação dos Componentes da Economia.}

Seja uma economia composta de $N$ consumidores, indexados por $i$; $M$ firmas, indexadas por $j$, e dois bens privados:

- trabalho fornecido pelo indivíduo $i\left(x^{i} \leq 0\right)$ ou utilizado pela firma $j\left(x^{j} \leq 0\right)$;

- bem privado produzido pela firma $j\left(y^{i} \geq 0\right)$ ou consumido pelo indivíduo $i\left(y^{i} \geq 0\right)$.

Em seu processo de produção cada firma gera um poluente $e^{i}(\leq 0)$ que afeta a qualidade do meio-ambiente ${ }^{5}$. A exemplo de outros autores, considera-se a emissão de poluente como um fator de produção ${ }^{6}$.

A eficiência na produção da firma $j$ é descrita pela função

$$
f^{j}\left(y^{j}, x^{j}, e^{j}\right)=0 \quad \forall j,
$$

que é contínua, côncava, bidiferenciável c

i) $\quad \frac{\partial f^{j}}{\partial y^{j}}=f_{y}^{j} \geq 0 \quad$ e $\quad \frac{\partial f^{j}}{\partial x^{j}}=f_{x}^{j}>0 \quad \forall j$ :

ii) $\forall y^{j} \exists\left(\hat{x}^{j}, \hat{e}^{j}\right)$ tal que

$$
-\frac{d x^{j}}{d c^{j}}=\frac{\partial f^{j} / \partial c^{j}}{\partial f^{j} / \partial x^{j}}=\frac{f_{e}^{j}}{f_{x}^{j}} \quad\left\{\begin{array} { l } 
{ > } \\
{ = 0 } \\
{ < }
\end{array} \quad \text { se } \quad e ^ { j } \left\{\begin{array}{l}
< \\
=e^{i} \forall j . \\
>
\end{array}\right.\right.
$$

\footnotetext{
5 A poluição em çuestão é a de origem industrial: e apenas os consumidores são afetados.

6 Esta é a abordagem tanbém adotada por Kílevorick c Kíramer (1979, p. 107) e Mallory (1990, p.25). 
iii) $y^{j}>0 \Longrightarrow x^{j}<0$.

A hipótese (ii) significa que a tecnologia admite, por um lado, a substituição entre os fatores de produção - o trabalho e as emissões - mas que, por outro lado, esta substituição não é sempre "útil". É suposto assim que, ceteris paribus, existe para o poluente um nível de emissão além do qual emitir mais significa empregar uma maior quantidade do fator trabalho. Esta condição está representada na Figura 1 .

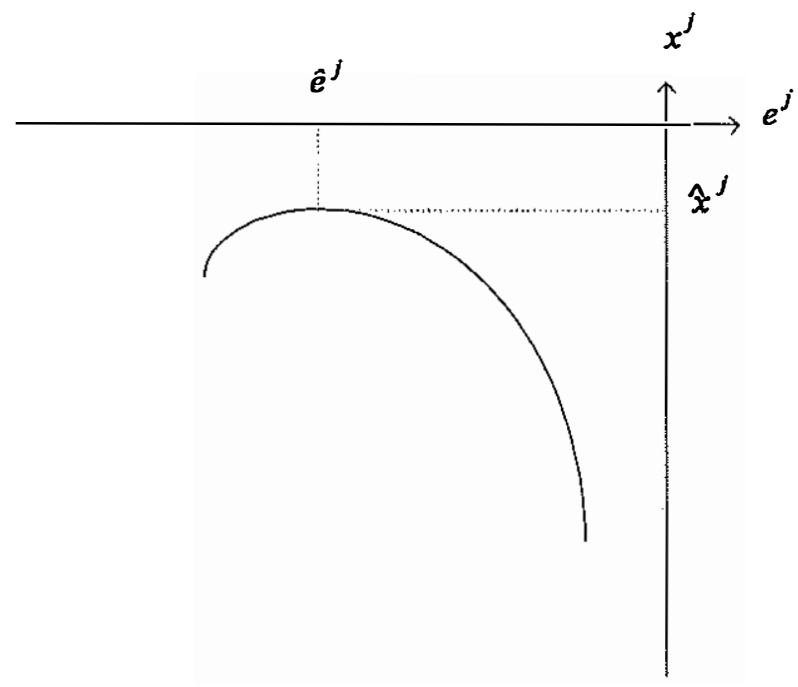

Figura 1: Isoquanta da Função de Proclução $f^{j}(\bullet)$

A região de interesse é limitada por $\hat{e}^{j} \leq c^{j} \leq 0, \forall j$ : se o input "meio-ambiente" é um bem livre (e o input trabalho é um recurso raro), as empresas vão utilizá-lo até e $\hat{e}^{j}, \forall j$. Ou seja, cada empresa tem todo 
objetivo de utilizar o mínimo de trabalho ${ }^{7}$. A hipótese (iii) assegura que o fator trabalho é necessário a toda atividade de produção.

No que concerne à relação entre emissões das firmas e qualidade do meio-ambiente, se admite que cada firma contribui diferentemente (o impacto depende de sua localização, das características do fuxo etc). Para considerar tal diferenciação, utiliza-se as funções denominadas na literatura sobre meio-ambiente como funşôes de transferência. Seja $q(\leq 0)$ o índice de qualidade ambiental. Então:

$$
q=q\left(e^{1}, \cdots, e^{J}\right) \quad \operatorname{com} \quad \frac{\partial q}{\partial e^{j}}=\delta^{j} \geq 0 \quad \forall j,
$$

sugerindo assim que um aumento das emissões de cada empresa pode conduzir a uma deterioração da qualidade ambiental. Tal relação permite quantificar o impacto de cada unidade de poluente emitido pela empresa $j$ sobre a qualidade do meio-ambiente ${ }^{8}$.

A qualidade ambiental afeta o bem-estar dos consumidores. $\mathrm{O}$ comportamento de cada um é descrito pela função utilidade ${ }^{9}$

$$
u^{i}\left(y^{i}, x^{\imath}, q\right) \quad \forall i
$$

que é estritanente côncava, contínua, bidiferenciável e

T Esta hipótese engloba o caso em que há urna relação constante entre o nivel de emissōes e o nivel de produção (como adotado pela naior parte dos trabalhos sobrc poluição, como por exemplo Kohn $(1985,1986))$, mas permite taubxem unk redução no nivel de poluição mantendo o nivel de produção constante. Ela é necessária para a existencia do equilibrio de desacordo, conforme será visto adiante.

$8 \Lambda$ linearidade implicada pela consteincia dos $\delta i \dot{e}$ evidentemente uma restrição na classe de funçõas de transferência. Sabese que os processos de poluição são melhor descritos por funções não lineares. Além disso, no caso de vários tipos de poluentes, há a possibilidacle de interação entre eles. 'Tulkens (1972, p.386) mostra que, neste caso, os resultados qualitativos não seriam afetados. De qualcuer modo, o exaune de formas mais gereus está fora dos objetivos deste trabalho.

9 Olxservat-se que a qualidade do nxio-ambiente entra no mesno nivel nas funçōes utilidade de todos os agrentes $i$ : $q$ é um bem público "without free disposal" (Milleron, 1972). 

i) $\frac{\partial u^{i}}{\partial y^{i}}=u_{y}^{i} \geq 0$
$\frac{\partial u^{i}}{\partial x^{i}}=u_{x}^{i}>0$
e $\quad \frac{\partial u^{i}}{\partial q}=u_{q}^{i} \geq 0$
$\forall i$
ii) $x^{i}=0 \Longrightarrow u_{y}^{i}=0$ e $u_{q}^{i}=0$.

Pode-se então definir:

- $\gamma_{e}^{j}=f_{c}^{j} / f_{x}^{j} \geq 0$ custo marginal de redução das emissões, em termos de trabalho;

- $\gamma_{y}^{j}=-f_{y}^{j} / f_{x}^{j} \geq 0$ custo marginal de produção do bem privado, em termos de trabalho;

- $\pi_{y}^{i}=u_{y}^{i} / u_{x}^{i} \geq 0$ avaliação subjetiva do bem privado pelo agente $i$;

- $\pi_{g}^{i}=u_{g}^{i} / u_{x}^{i} \geq 0$ avaliação subjetiva da qualidade do meioambiente pelo agente $i$.

\section{Estados da Economia.}

Um estado da economia é representado pelo vetor $s$, cujos elementos são os planos de consumo dos indivíduos e os planos de produção das empresas.

Um estado sé realizúvel se $\left(y^{i}, x^{i}, q\right) \in X^{i}$ (conjunto de consumo) $\forall i,\left(y^{j}, x^{j}, e^{j}\right) \in Y^{j}$ (conjunto de produção) $\forall j, \mathrm{e}$

$$
\begin{aligned}
& \sum_{j} y^{j}=\sum_{i} y^{i} \\
& \sum_{j} x^{j}=\sum_{i} x^{i}
\end{aligned}
$$


Politica Ambiental

$$
q=q\left(e^{1}, \cdots, e^{J}\right)
$$

As equações (3) e (4) representam o equilíbrio entre oferta e demanda do bem privado e do trabalho, respectivamente.

Uim estados é eficiente zável e se não cxistc um outro estado $\hat{s}$ realizável utilidade de um indivíduo seja maior, sem que a de um outro tenha decrescido.

As condições do eficiência para esta economia podem ser obtidas maximizando-se a utilidade de cada agente $i$ sob as restrições (1), (3)-(5) e sob a condição que a utilidade dos outros agentes não sejam reduzidas ${ }^{10}$. Como resultado, obtém-se:

Condição 1: Se $s$ é um estado ESP, então $\pi_{y}^{i}=\gamma_{y}^{j} \quad \forall i, j$.

Esta é a condição clássica para um bem privado: em um estado ESP. o custo marginal de produção do bem privado para a firma $j$ é igual à disponibilidade marginal do agente $i$ a pagar por este bem.

Condição 2: Para todo estado ESP, $\sum_{i} \pi_{y}^{i}=\gamma_{c}^{j} / \delta^{j} \quad \forall j$.

Tem-se aqui a famosa condição de Samuelson para a provisão de um bem público: em um estado ESP, o conjunto de todos os consumidores está disposto a pagar (na margem), para ter uma melhor qualidade ambiental, um valor igual ao que cada firma aceita para reduzir suas descargas (na margem).

\section{A Economia Descentralizada.}

A fim de descentralizar esta economia se supõe que cada consumidor (restrito por seu orçamento) maximiza sua utilidade e que cada firma maximiza seu lucro (sob a restrição da tecnologia disponível).

10 Para uma formalização do problema, ver Raunos (1992). 
Portanto, seja $\tau$ o preço do bem privado, onde o trabalho é o numerário. Pode-se definir um equilíbrio competitivo onde as firmas são livres para poluir e a única ação dos consumidores é de se ajustar à qualidade do meio-ambiente:

Equilíbrio de desacordo ${ }^{11}$ : é dado pelo sistema de preços $(\tau, 1)$ e pelas atividades de consumo e de produção tais que:

i) $\left(y^{i}, x^{i}, \bar{q}\right) \max u^{i}\left(y^{i}, x^{i}, \bar{q}\right)$ sujeito a $\tau y^{i}+x^{i}=\alpha_{i} \sum_{j} B^{j} \quad \forall i$, $\alpha_{i} \geq 0$ e $\sum_{i} \alpha_{i}=1$;

ii) $\left(y^{j}, x^{j}, e^{j}\right) \max B^{j}=\tau y^{j}+x^{j}$ dada a restrição tecnológica (1);

iii) as equações (3)-(5) sejam satisfeitas.

Vê-se que a Condição 1 é satisfeita: $\pi_{y}^{2}=\tau=\gamma_{y}^{j} \quad \forall i, j$. Para o bem público este equilíbrio fornece, no que concerne às firmas, $\gamma_{e}^{j}=$ $0 \quad \forall j$. Assim, este equilíbrio é ESP se e somente se $\pi_{q}^{i}=0 \quad \forall i$. Portanto, se para pelo menos um agente $i$ tem-se que $\pi_{q}^{i} \neq 0$, então não se está em um estado ESP. Para corrigir isto, adota-se a sugestão de Pigou (1932) e Baumol (1972): uma autoridade ambiental impõe uma $\operatorname{taxa} \theta$ por unidade emitida. A fim de redistribuir a receita (déficit) da taxa, utiliza-se pagamentos compensatórios $t^{i}$. Portanto, define-se:

Equilibrio Pigou-Baumol (PB): é representado por um sistema de preços $(\tau, 1)$, de taxas sobre as emissões $\theta$, de pagamentos compensatórios $t$ e pelas atividades de consumo e de produção tais que:

11 Conceito proposto por Tulkens e Schoumaker (1975) para uma economia com poluidores mas sem produção. 
i) $\left(y^{i}, x^{i}, \bar{q}\right) \max u^{i}\left(y^{i}, x^{i}, \bar{q}\right)$ sujeito a

$$
\tau y^{i}+x^{i}+t^{i}=\alpha_{i} \sum_{j} B^{j} \quad \forall i
$$

ii) $\left(y^{j}, x^{j}, e^{j}\right) \max B^{j}=\tau y^{j}+x^{j}+\theta \delta^{j} e^{j}$ sob a restrição (1);

ii) equações (3)-(5) sejam satisfeitas.

A Condição 1 ainda é satisfeita. No que concerne a qualidade do meio-ambiente, verifica-se que cada firma produz onde $\gamma_{c}^{j} / \delta^{j}=\theta$ $\forall j$, qualquer que seja o nível de $\theta$. Quantio a $q$, ele é exógeno às ações do consumidor e determina o valor de $\pi_{q}^{2}$.

Vale ressaltar que um equilíbrio PB é da mesma natureza que um equilíbrio com relação a um sistema de preços e de rendas no sentido de Malinvaud (1972), em que a renda dos consumidores é determinada por suas dotações iniciais, suas partes nos lucros das firmas e as transferências compensatórias provenientes da redistribuição da receita da taxa. Note-se ainda que o equilíbrio de desacordo é um equilíbrio $\mathrm{PB}$ para o qual $\theta=0$ e $t^{i}=0$ para todo $i$. Além disso, a Condição 2 é satisfeita se e somente se $\theta=\sum_{i} \pi_{q}^{i}$, isto é, se a soma das taxas marginais de substituricäo é igual a taxa sobre as emissóes. Sob esta condição suplementar, um equilíbrio PB é ESP. Ressalte-se ainda que a receita da taxa é inteiramente distribuída aos consumidores; de fato, a soma das restrições orçamentárias para todos os agentes permite obter $\sum_{i} t^{i}=\sum_{j} \theta \delta^{j} e^{j}$.

\section{O Ajustamento Dinâmico.}

Pode-se resumir o acima exposto: a situação de um equilíbrio de desacordo é aquela de um equilíbrio $\mathrm{PB}$ onde $\theta=0$ e as empresas não pagam pela utilização do ambiente, ou seja, o recurso "meio-ambiente" é considerado como um bem livre. Niesta situação nenhuma ação coletiva é tomada para controlar as externalidades. 
Sabe-se que um equilíbrio PB tem por propriedade que $\theta=\gamma_{\mathfrak{c}}^{j} / \delta^{j}$ para todo $j$, ou seja, uma vez que a autoridade ambiental defina o valor da taxa sobre as emissões, as firmas atuarão onde seus custos marginais de redução das emissões iguale este valor. Sabe-se ainda que a eficiência Paretiana é obtida onde

$$
\sum_{i} \pi_{q}^{i}=\theta
$$

Caso esta última igualdade não seja satisfeita, pode-se utilizar a informação fornecida pela diferença entre os dois membros da expressão acima a fim de escrever a regra de ajustamento seguinte ${ }^{12}$ :

$$
\dot{\theta}=\sum_{i} \pi_{q}^{i}-\theta
$$

ou seja, a taxa deve ser aumentada se a avaliação agregada da qualidade do ambiente feita pelos consumidores ultrapassa, na margem, o valor atual da taxa e vice-versa ${ }^{13}$. Como conseqüência da variação da taxa, as firmas ajustam suas emissões e o montante de pagamento para cada uma varia de

$$
\delta^{j}\left(\dot{\theta} e^{j}+\theta \dot{e}^{j}\right) \quad \forall j .
$$

Dada a variação no "preço" da qualidade do meio-ambiente, cada firma ajusta suas emissões para baixo se este "preço" ultrapassa o custo marginal de redução de poluição, e vice-versa. Tem-se assim que:

$$
\dot{e}^{j}=\theta-\gamma_{e}^{j} / \delta^{j} \quad \forall j
$$

12 Considera-se que o parâmetro de velocidade de ajuste é normalizado a 1.

$13 \dot{E}$ assumido que os consumidores revelam corretarmente à autoridade ambiental as suas disposiçōes naarginais a pagar por uma melhoria na qualidade do meio-ambiente. 
o que implica em uma modificação na qualidade do meio-ambiente, e a equação (5) fornece

$$
\dot{q}=\sum_{j} \delta^{j} \dot{e}^{j}
$$

No que concerne ao bem privado, se utiliza o modelo de ajuste proposto por Tulkens e Zamir (1979, seção 5.2) segundo o qual quando o consumidor está frente a um sistema de preços e possui uma cesta de bens que não é uma cesta de equilíbrio, ele realoca seu orçamento de forma a maximizar a variação de sua utilidade. Em termos infinitesimais, esta realocação é dada por

$$
\dot{y}^{i}=\pi_{y}^{i}-\tau \quad \forall i .
$$

No mesmo espírito que para os consumidores, a produção do bem privado é modificada segundo a diferença entre o custo marginal de produção do bem e seu preço:

$$
\dot{y}^{j}=-\left(\gamma_{y}^{j}-\tau\right) \quad \forall j,
$$

onde o preço do bem privado é dado pela média das disposições marginais a pagar dos consumidores e firmas:

$$
\tau=\frac{1}{M+N}\left(\sum_{i} \pi_{y}^{i}+\sum_{j} \gamma_{y}^{j}\right)
$$

Resta determinar como variam as quantidades utilizadas e ofertadas de trabalho. No que se refere à quantidade utilizada, a função de produção (1) permite obter a variação desta quantidade como função da variação da quantidade do bem privado e do nível das emissões:

$$
\dot{x}^{j}=-\gamma_{y}^{j} \dot{y}^{j}-\gamma_{e}^{j} \dot{e}^{j} \quad \forall j .
$$


Quanto a quantidade ofertada por cada indivíduo, utiliza-se a equação (2) para se obter a variação das utilidades:

$$
\dot{u}^{i}=u_{x}^{i}\left(\pi_{y}^{i} \dot{y}^{i}+\dot{x}^{i}+\pi_{q}^{i} \dot{q}\right) \quad \forall i
$$

e a equação (6) para se obter a variação da quantidade de trabalho fornecida por cada indivíduo:

$$
\dot{x}^{i}=-\dot{r} y^{i}-\tau \dot{y}^{i}-\dot{t}^{i}+\alpha_{i} \sum_{\jmath} \dot{B}^{i} \quad \forall i .
$$

A substituição de (16) em (15) permite chegar a

$$
\begin{aligned}
\dot{u}^{i} & =u_{x}^{2}\left[\left(\dot{y}^{i}\right)^{2}+\alpha_{i}\left(\dot{y}^{j}\right)^{2}+\alpha_{i} \sum_{j} \delta^{j}\left(\dot{e}^{j}\right)^{2}+\pi_{q}^{i} \dot{q}\right. \\
& \left.-\dot{\tau}\left(y^{i}-\alpha_{i} \sum_{j} y^{j}\right)+\alpha_{i} \sum_{j} \delta^{j} \dot{\theta}^{j} e^{j}-\dot{t}^{i}\right]
\end{aligned}
$$

$\forall i$.

Pode-se então concluir que se a variação das transferências compensatórias é dada por:

$$
\begin{aligned}
\dot{t}^{i} & =\pi_{q}^{i} \dot{q}-\alpha_{i} \sum_{j} \delta^{j} \theta \dot{e}^{j}-\dot{\tau}\left(y^{i}-\alpha_{i} \sum_{j} y^{j}\right) \\
& +\alpha_{i} \sum_{j} \delta^{j}\left(\dot{\theta} e^{j}+\theta \dot{e}^{j}\right) \quad \forall i,
\end{aligned}
$$

a monotonicidade do processo é assegurada. Pode-se fornecer uma interpretação da regra (17):

- o primciro termo é consistente com o "benefit principle" da teoria das finanças públicas: a cada indivíduo é solicitado uma contribuição para a melhoria da qualidade do meio ambiente que é proporcional ao "valor" deste bem; 
- o segundo termo é um complemento do primeiro: ele leva em consideração a contribuição de cada indivíduo através das empresas;

- o terceiro considera a variação dos preços dos bens privados: o indivíduo é compensado em proporção à diferença entre o que ele consome e o que ele dispõe;

- o quarto termo é uma compensação para o indivíduo $i$, devido as variações da taxa e das emissões das empresas, proporcional a sua parte de propriedade nas mesmas.

Em resumo, o processo dinâmico com taxas pigouvianas é dado por (8)-(14) e (16)-(17), tendo como condições iniciais aquelas de um equilíbrio Pigou-Baumol para o qual $\theta=0$ e $t^{i}=0 \quad \forall i$, que caracteriza um equilíbrio de desacordo.

\section{Propriedades do Processo.}

O processo definido pelas equações (8)-(14) e (16)-(17):

a) é fisicamente possível;

b) é individualmente racional;

c) converge para um estado ESP.

Prova:

a) A soma de (11) para todos os consumidores e de (12) para todas as firmas conduz, com a utilização de (13), a $\sum_{i} \dot{y}^{i}=\sum_{j} \dot{y}^{j}$. Isto implica que (3) é verificada. Repetindo o mesmo argumento para (14) e (16) obtém-se que (4) é satisfeita. 
b) A substituição de (17) e (16) em (15) permite chegar a

$$
\dot{u}^{i}=u_{x}^{i}\left[\left(\dot{y}^{i}\right)^{2}+\alpha_{i} \sum_{j}\left(\dot{y}^{j}\right)^{2}+\alpha_{i} \sum_{j} \delta^{j}\left(\dot{e}^{j}\right)^{2}\right] \geq 0 \quad \forall i
$$

Esta expressão se anula apenas no ponto de ótimo. Em qualquer outro ponto, pelo menos um dos indivíduos tem interesse em que o processo se realize, e os outros não têm qualquer objeção em participar: seus níveis de utilidade não serão reduzidos.

c) Para a convergência, Champsaur-Drèze-Henry (1977) estabelece que se um sistema de equações diferenciais tem uma solução ${ }^{14}$ e se existe uma função de Lyapunov para tal sistema, então esta solução é quase-estável. Este é exatamente o caso analisado aqui, já que a função de Lyapunov pode ser denotada por $W(t)=\sum_{i} \lambda_{i} u^{i}(t)$. Dado que o único ponto onde $\dot{u}^{i}$ é zero é no ótimo, o sistema converge para um estado ESP.

\section{Ajustamento dos Preços.}

A equação (13) permite descrever a variação do preço do bem privado por

$$
\dot{\tau}=\frac{1}{M+N}\left(\sum_{i} \dot{\pi}_{y}^{i}+\sum_{j} \dot{y}_{y}^{j}\right) .
$$

Esta expressão denota uma forma particular de ajuste do preço do bem privado sobre seu mercado. Esta forma de ajuste que acompanha a sucessão das alocações realizáveis determinadas pelo processo foi proposta em Tulkens e Zamir (1979, seção 5.2) e desenvolvida em Chander e Tulkens (1991) no quadro de economias de

14 Para as dernonstraçōes de existência de uma solução para este processo (tâtonnement e não. tâtonnement) ver Henry (1972), Tulkens (1979, p.209), e d'Aspremont e Tulkens (1980, p.398). 
troca pura. Estes autores a deduziram de uma teoria de formação dos preços que repousa sobre considerações da Teoria dos Jogos. Ela é aplicada aqui ao caso de uma economia com produção. Vale a pena ressaltar, como mencionam Chander e Tulkens (1991, p.16), que este modo de ajuste se aparenta a teoria do non-tâtonnement (Negishi, 1962) - ou ainda ao processo de arbitragem de Allais (Allais, 1967).

\section{Conclusão.}

A questão de como corrigir as externalidades negativas causadas pela poluição tem gerado uma enorme discussão na literatura. Esta discussão

dentro de um elenco que vai desde os primitivos limites sobre as emissões das firmas, até a criação de mercados artificiais. De uma maneira geral, os trabalhos partem da constatação da ineficiência de uma economia laissez-faire e, apoiados num conceito-solução, determinam qual a situação ótima para a economia e de que forma intervir para restaurar a eficiência.

A preocupação neste trabalho vai um pouco mais além: caracterizada uma situação não ótima, e adotando o conceito-solução de eficiência de Pareto, questiona-se se é possível, partindo do primeiro, chegar ao segundo. C'aso afirmativo, indaga-se se o sistema tende a voltar ao estado de equilíbrio quando submetido a perturbações. Por fim, pergunta-se se é possível interromper o processo a qualquer momento (ou seja, se é um processo de non-tãtonnement). Mostrou-se que com a adoşão de una política ambiental em que se utiliza um instrumento incitativo - as taxas Pigouvianas - é possivel responder afirmativamente às questões acima. Para isto, adotou-se a metodologia da análise de estabilidade.

A implementação deste modelo, conforme explicitado ao longo de sua construção, tem algumas condicionantes. Talvez a mais forte delas se refira ao fato de que é necessário que os consumidores revelem suas verdadeiras disposições marginais a pagar. Na literatura sobre provisão de bens públicos há diversos trabalhos que discutem esta 
questão, como por exemplo, Roberts (1979).

Uma outra condicionante se refere à estrutura contínua do processo. Sabe-se que a planificação real na economia se faz em tempo discreto e se atinge um final após algumas iterações. Logo, é mais realista supor que as ações destinadas a efetuar variações na qualidade ambiental se produzem em tempo discreto. Assim, após se estabelecer uma direção de variação, tais ações possibilitam levar em consideração o período de tempo que permite a todas as outras variáveis se ajustarem. Em seguida, novas modificações podem ser consideradas e implementadas. Portanto, a elaboração de um processo em tempo discreto é a próxima etapa na construção de tais modelos.

Submetido em Dezembro de 1994. Revisado em Setembro de 1995.

\section{Referências}

Allais, M. 1967. "Les conditions de l'efficacité dans l'économie." IV Seminario Internazionale, Rapallo, Centro Studi e Richerche sui Problemi Economico-Sociali, CESE, Milan.

Baumol, W.J. 1972. "On taxation and the control of externalities." American Economic Review 62: 307-322.

Baumol, W.J. \& Oates, W.E. 1988. The Theory of Environmental Police. Cambridge: Cambridge University Press.

Bohm, P. \& Russell, C.S. 1985. "Comparative analysis of alternative policy instruments." In A.V. Inneese e J.L. Sweeney eds., Handbook of Natural Resource and Energy Economics, Vol. I, cap. 10. Elsevier Science Publishers.

Champsaur, P., Drèze, J.H. \& Henry, C. 1977. "Stability theorems with economic applications." Econometrica 45: 273-294.

Chander, P. \& Parikh, A. 1990. "Theory and practice of decentralized planning procedures." Journal of Economic Surveys 4: 19-58. 
Chander, P. \& Tulkens, H. 1990. "Exchange processes, the core and competitive allocations." In J.J. Gabszewicz, J.F. Richard and L.A. Wolsey eds., Economic Decision-Making: Games, Econometrics and Optimization: Contributions in Honour of Jacques H. Drèze. Amsterdam: North-Holland.

d'Aspremont, C. \& Tulkens, H. 1980. "Commodity exchanges as gradient processes." Econometrica 48: 387-399.

Drèze, J. \& de la Vallèe-Poussin, D. 1971. "A tâtonnement process for public goods." Revieus of Economic Studies 37: 133-150.

Henry, C. 1972. "Differential equations with discontinuous righthand side for planning process." Journal of Economic Theory 4: 545-551.

Klevorick, A.K. e Kramer, G.H. 1973. "Social choice on pollution management: the Genossenschaften." Journal of Public Economics 2: 101-146.

Kohn, R. 1985. "A general equilibrium analysis of the optimal number of firms in a polluting industry." Canadian Journal of Economics 17.

Kohn, R. 1986. "The rate of emission and the optimal scale of the polluting firm." Canadian Jou,rnal of Economics 19.

Malinvaud, E. 1970. "Procedures for the determination of a program of collective consumption." European Economic Review 2: 187-217.

Malinvaud, E. 1971. "A planning approach for the public good problem." Swedish Journal of Economics 73: 96-112.

Malinvaud, E. 1972. "Prices for individual consumption, quantity indicators for collective consumption." Review of Economic Studies 39: 385-406.

Mallory, C.D. 1990. "A non-tâtonnement process for an economy with water pollution." Tese de Doutorado. Universidade de Montreal, Canada. 
Milleron, J.-C. 1972. "Theory of value with public goods: a survey article." Journal of Economic Theory 5: 419-472.

Negishi, T. 1962. "The stability of competitive economy: a survey article." Econometrica.

Pigou, A.C. 1932. The Economics of Welfare. Londres: MacMillan and Co.

Ramos, F.S. 1992. "Pigovian taxes, tradable permits and a dynamic process for an economy with pollution." In J.W. Owsinski, J. Stefanski e A. Straszak eds., Transition to Advanced Market Economies, 445-455. Varsovia: Association of Polish Operational Research Societies.

Roberts, J. 1979. "Incentives in planning procedures for the provision of public goods." The Review of Economic Studies 46: 283-292.

Tulkens, H. 1972. "Traitement au moindre coût des effluents industriels et tarification d'une station d'épuration." Recherches Economiques de Louvain 4-5.

Tulkens, H. 1978. "Dynamic process for public goods: an institution-oriented survey." Journal of Public Economics 9: 163-201.

Tulkens, H. 1979. "An economic model of international negotiation relating to transfrontier pollution." In K. Krippendorf ed., Communication and Control Society, 199-212. Gordon and Breach Science Publishers.

Tulkens, H. \& Schoumaker, F. 1975. "Stability analysis of an effluent charge and the polluters pay principle." Journal of Public Economics 4: 245-269.

Tulkens, H. \& Zamir, S. 1979. "Surplus-sharing local games in dynamic exchange process." Review of Economic Studies 46: 305-313. 
1

1

1

1

I

1

1

1

1 